\title{
Impact of Needle and Syringe Program Coverage on HIV Incidence in People Who Inject Drugs
}

\author{
Mehdi Noroozi, ${ }^{1}$ Alireza Noroozi, ${ }^{2,3}$ Ali Mirzazadeh, ${ }^{4,5}$ Ahmad Hajebi, ${ }^{6}$ Yadoallah Mehrabi, ${ }^{7}$ Sayed \\ Saeed Hashemi, ${ }^{7}$ Hamid Sharifi, ${ }^{5}$ Seyed Ebrahim Ghoddousi, ${ }^{8}$ Korosh Etemad, ${ }^{7}$ and Hamid Soori ${ }^{9,}$ \\ ${ }^{1}$ Substance Abuse and Dependence Research Center, University of Social Welfare and Rehabilitation Sciences, Tehran, IR Iran \\ ${ }^{2}$ School of Advanced Technologies in Medicine (SATiM), Tehran University of Medical Sciences (TUMS), Tehran, IR Iran \\ ${ }^{3}$ Substance Abuse Prevention and Treatment Office (SAPTO), Ministry of Health and Medical Education (MoHME), Tehran, IR Iran \\ ${ }^{4}$ Department of Epidemiology and Biostatics, University of California, San Francisco, CA, USA \\ ${ }^{5}$ Regional Knowledge Hub, and WHO Collaborating Centre for HIV Surveillance, Institute for Futures Studies in Health, Kerman University of Medical Sciences, Kerman, IR \\ Iran \\ ${ }^{6}$ Mental Health Research Center, Tehran Institute of Psychiatry, Faculty of School of Behavioral Sciences and Mental Health, Iran University of Medical Sciences, Tehran, IR Iran \\ ${ }^{7}$ Department of Epidemiology, Safety Promotion and Injury Prevention Research Center, Shahid Beheshti University of Medical Sciences, IR Iran \\ ${ }^{8}$ Mental Health, Social Health and Addiction Department (MeHSHAD), Substance Abuse Prevention and Treatment Office (SAPTO), Ministry of Health and Medical Education, \\ Tehran, IR Iran \\ ${ }^{9}$ Safety Promotion and Injury Prevention research center, School of Public Health, Shahid Beheshti University of Medical Sciences, Tehran, Iran \\ "Corresponding author: Hamid Soori, Department of Epidemiology, Safety Promotion and Injury Prevention Research Center, Shahid Beheshti University of Medical Sciences, \\ IR Iran. Tel/Fax: +98-2122431993, E-mail: hsoori@yahoo.com
}

Received 2015 March 30; Revised 2015 September 14; Accepted 2016 February 21.

\begin{abstract}
Background: The sharing of syringes by people who inject drugs (PWID) is an important factor for the transmission of HIV. Needle and syringe programs (NSP) are widely used to reduce any harm associated with drug injection, however since the impact of these programs have not been systematically studied our objective is to model the impact of NSPs on HIV incidences for PWID in Kermanshah, Iran.

Objectives: The aim of this study was to determine the effectiveness of NSP coverage in prevention of HIV incidence among PWIDs. Patients and Methods: We used Wilson et al.'s mathematical model to predict the occurrence of HIV amongst PWID with sufficient and insufficient client-level coverage of NSPs. We parameterized and calibrated the model using behavioral and epidemiological data collected in an empirical study of 470 active PWID and recruited them through two Drop-In Centers (DIC) in Kermanshah, Iran from April to September, 2014. Other parameters such as risk of HIV transmission through injection with a shared injection, and effectiveness of syringe cleaning were obtained from the literature. We applied Monte Carlo simulation (10,000 runs) to capture the uncertainty (simulation interval-SI) in the model's output.

Results: Given the output of the model, we found that among the PWID with sufficient coverage of NSPs the HIV rate is $1.02 \%$, while those with insufficient coverage is increased to $4.04 \%$ (risk different $=3 \%$, SI 95\% $2.7-3.4 \%$ ). By reducing the percentage of sharing from $18 \%$ (in patients with insufficient NSPs coverage) to 10\%, the HIV incidence will be Dropped to 0.9\% (SI 95\%, 0.4 - $1.3 \%$ ).

Conclusions: We found a large impact due to NSPs for reducing the occurance of HIV among active drug injectors who have been provided sufficient needles and syringes. The coverage of NSPs needs to be increased to observe such significant impact.
\end{abstract}

Keywords: Mathematical Model, People Who Inject Drugs (PWID), Effectiveness

\section{Background}

It is estimated that about 12.7 million people inject drugs globally, and 1.7 million (13\%) of those are living with HIV (1). The significant spread of HIV infection amongst people who inject drugs (PWID) has been shown in many countries such as Europe, Asia and North America $(2,3)$. In Iran, there are approximately 170,000 - 230,000 PWID of whom, $15 \%$ are infected with $\operatorname{HIV}(4,5)$. Over two-thirds of all new identified HIV cases have been attributed to unsafe injection (4, 6-8). To reduce the risk and harms associated with injections, needle and syringe programs (NSP) have been developed and implemented widely (9-12). In
Iran, NSP is the cornerstone of HIV prevention interventions and has been implemented in different community settings since $2002(8,10,13-15)$. NSP is being delivered through drop-in centers, (DIC) for those who have access to services and by outreach and second-hand exchanges (7, 8). The DIC is a place that addresses the health needs of PWID. Additionally, it supports the rights of people using drugs for treatment and care services that are respectful and non-discriminatory. The DIC also functions as a fixed outlet for a needle and syringe exchange program (NSEP), where in these programs they can exchange their used needles for new ones at the DIC. These centers and outreach 
needle and syringe programs provide sterile needle and syringe services, deliver training on safe injecting practices on overdose prevention as well as providing condom and safe sex education (16). The effectiveness of these programs have been studied and reported to be an effective intervention in developed countries $(9,17-20)$, however the effect of NSP with different coverage was not studied in Iran. The number of needles and syringes given to a person who injects drugs should be corresponded with his or her frequency of injection; otherwise, the effect of this program may be compromised or even be harmful (21). In this study, our hypothesis states that low NSP coverage (insufficient proportion of all drug injections becoming safe through distributed syringes) will not effectively reduce the harms associated with the injection and so associated with high transmission of HIV amongst PWIDs. Using empirical data collected from two drop-in centers and an outreach team in Kermanshah, Iran as well as a mathematical model, we assessed the impact of insufficient NSP on HIV incidences among PWID.

\section{Objectives}

The aim of this study was to determine the effectiveness of NSP coverage in prevention of HIV incidence among PWIDs.

\section{Patients and Methods}

\subsection{Study Design}

This was a model study to predict the annual incident of HIV amongst PWID with sufficient and insufficient client-level coverage of NSPs. To explore the expected national annual incidence associated with different levels of sterile syringe distribution we used a mathematical model, which was designed by Wilson and colleagues (21). Sufficient needles and syringes defined to potentially have one clean needle/syringe available per injection and were estimated from frequency of injection and syringes pick up. The input data used for our mathematical model was collected by a self-reported interview-based questionnaire completed by 470 PWIDs in Kermanshah, Iran. Study participants were recruited from the community and NSP sites between September and December 2014. PWID from NSP sites were recruited at the facility by convenience sampling; where as community-based people were recruited by outreach, random street-walk and peer-referral (snowball using referral coupons) sampling. People who met the eligibility criteria were approached and asked to participate in our study after providing informed consent. The criteria to become eligible and be recruited into the study was that the male clients were 18 years of age or older, reported drug injection within the last month prior to the interview, and gave written consented to participate in the study. The study protocol and all the procedures were reviewed and approved by the ethical committee of the Kerman University of Medical Sciences (ethical code k/93/204). At the DIC, a trained male psychologist introduced the study objectives, explained the risk and benefits of participation in the study as well as assessing the eligibility criteria. Next, the psychologist interviewed each consented individual using a standardized structured questionnaire based on the client-level coverage. Participants were then divided into two groups: those with the coverage less than $100 \%$ (insufficient coverage) and those with the coverage of $100 \%$ or more (sufficient coverage). We used a deterministic model to explore the expected annual occurrence of HIV associated with the different coverages of NSP. The expected number of new HIV infections (I) among people who inject drugs was estimated by the Equation 1:

$I=\frac{N n s q \delta \beta(1-\rho \times \in)}{m^{2}} \sum\left(\frac{m}{r}\right) \rho^{r}(1-p)^{m-r} \frac{r m-r^{2}}{r+1}$

$\mathrm{N}$ : number of PWID in each group; n: number of injections per year for each PWID; S: proportion of IDUs who share syringes; q: proportion of their injections may shared; $r$ : infected people in a sharing group; p: initial prevalence HIV among PWID; $\rho$ : proportion that cleaning syringe occurs before sharing injections; $\delta$ : average number of times each shared syringe is used before disposal; $\beta$ : HIV transmission probability per injection with contaminated syringe; $\varepsilon$ : effectiveness of syringe cleaning.

\subsection{Model's Parameters}

We parameterized the model using behavioral and epidemiological data collected in an empirical study of PWID. Using the empirical data, in those PWID with and without sufficient NSP coverage, we estimated the proportion of people who inject drugs may share their syringes (p); proportion of their injections they may share (q), average size sharing groups $(\mathrm{m})$ and HIV prevalence amongst PWID $(\mathrm{P})$. Other biological parameters such as risk of HIV transmission per injection with a shared injection $(\beta)$, and effectiveness of syringe cleaning to prevent virus transmission $(\varepsilon)$ were obtained from literature (22-30). A summary of such parameters for each group, with and without sufficient NSP coverage, is presented in Table 1.

\subsection{Estimation of HIV Averted}

To capture the uncertainty in the model parameters, we applied a Monte Carlo simulation model. This was to 
Table 1. Model Input Parameters and Distribution Types Used in Uncertainty Analysis in two Groups

\begin{tabular}{|c|c|c|c|c|}
\hline \multirow{2}{*}{$\begin{array}{l}\text { Input Parameters (PWID } \\
\text { Reported Behavior) }\end{array}$} & \multirow[t]{2}{*}{ Source of Data } & \multirow{2}{*}{$\begin{array}{c}\text { Probability Distribution } \\
\text { (Model Inputs) }\end{array}$} & \multicolumn{2}{|c|}{ Model Inputs for } \\
\hline & & & Access Insufficient $(\mathbf{n}=\mathbf{2 5 0})$ & Access Sufficient $(\mathrm{n}=\mathbf{2 2 0})$ \\
\hline $\begin{array}{l}\text { Average size of a sharing } \\
\text { group in each party }\end{array}$ & $\begin{array}{l}\text { Estimated from Kermanshah } \\
\text { Survey (9) }\end{array}$ & Triangular $(2-3.4)$ & 2.33 & 2.21 \\
\hline $\begin{array}{l}\text { HIV transmission } \\
\text { probability per injection } \\
\text { with contaminated syringe }\end{array}$ & Published literature (22-24) & Triangular (0.003-0.01) & 0.007 & 0.007 \\
\hline $\begin{array}{l}\text { Effectiveness of syringe } \\
\text { cleaning to prevent virus } \\
\text { transmission }\end{array}$ & Published literature $(25,26,30)$ & Triangular (0.4- 0.6) & $50 \%$ & $50 \%$ \\
\hline $\begin{array}{l}\text { Average number of } \\
\text { injections per PWID per year }\end{array}$ & $\begin{array}{l}\text { Estimated from Kermanshah } \\
\text { Survey (9) }\end{array}$ & Triangular (650 - 2200 & 1180 & 1080 \\
\hline $\begin{array}{l}\text { Proportion of PWID who } \\
\text { share syringes }\end{array}$ & $\begin{array}{l}\text { Estimated from Kermanshah } \\
\text { Survey (9) }\end{array}$ & Triangular $(0.1-0.2)$ & $18 \%$ & $10 \%$ \\
\hline $\begin{array}{l}\text { Proportion of injections } \\
\text { that are shared by PWID per } \\
\text { year }\end{array}$ & $\begin{array}{l}\text { Estimated from Kermanshah } \\
\text { Survey (9) }\end{array}$ & Triangular (0.01- 0.5) & $0.1 \%$ & 0.06 \\
\hline $\begin{array}{l}\text { Average no. of times each } \\
\text { shared syringe is used } \\
\text { before disposal per year }\end{array}$ & $\begin{array}{l}\text { Estimated from Kermanshah } \\
\text { Survey (9) }\end{array}$ & Triangular (1-4) & 2.66 & 2.12 \\
\hline $\begin{array}{l}\text { Initial prevalence of HIV } \\
\text { among PWID }\end{array}$ & $\begin{array}{l}\text { Estimated from Kermanshah } \\
\text { Survey (9) }\end{array}$ & $\operatorname{Beta}(0.1-0.25)$ & $15 \%$ & $15 \%$ \\
\hline $\begin{array}{l}\text { Proportion of syringes } \\
\text { cleaned before reusing }\end{array}$ & $\begin{array}{l}\text { Estimated from Kermanshah } \\
\text { Survey (9) }\end{array}$ & Triangular $(0.2-0.9)$ & $47 \%$ & $60 \%$ \\
\hline
\end{tabular}

assess how the uncertainty in the input parameters affects the overall impact estimates. To do this, for each model input we developed a probability distribution of the potential range of values that it could take (Table 1 ). We used the distribution of data we observed in the empirical study as the uncertainty measure. For others parameters, we used the literature to assign the proper distribution. Then, we ran the Monte Carlo simulation with 10,000 draws in two scenarios, first using the parameters for PWID with sufficient NSP coverage and then for PWID without sufficient NSP coverage. The estimated HIV annual incidence was reported as the main output of the model with a simulation interval (SI 95\%). We considered the relative risk reduction (RRR) of HIV incidences (HIV averted) as the NSP effectiveness measure. Furthermore, we conducted a probabilistic one-way sensitivity analysis on every single parameter to assess its effect on HIV occurrences. The effect was reported as tornado plot. We used the Ersatz software (http://www.epigear.com; Monte Carlo add-in program for MS Excel) for modeling and calculation.

\section{Result}

4.1. Characteristics of Study Participants in Kermanshah Survey A total of 470 men who injected drugs participated in the study; the mean age \pm SD was $33.8 \pm 8.58$ years. The majority of participants were unemployed (64\%), had a monthly income less than $\$ 150$ (85\%) and homeless (42\%). Concerning their HIV status, $16 \%$ did not know their serostatus and $15 \%$ of them were reported as positive. The mean for first time drug usage was $21.2 \pm 11.5$ years of age and the mean for first time drug injections was $26.7 \pm 12.5$ years of age.

\subsection{Injection-Related High-Risk Behaviours}

As reported in Table 1 , subjects reported a higher average number of injection per week (21.28 \pm 15.11 vs. 7.74 $\pm 7.58)$, sharing injections per week (3.10 \pm 5.42 vs. $0.4 \pm$ $0.97)$ and shared person in each party (2.33 \pm 2.0 vs. $2.37 \pm$ 0.80 ) in insufficient group, compared to sufficient group, all differences were statistically significant $(\mathrm{P}<0.001)$. Regarding the cleaning of the syringe before it is reused, $47 \%$ of subjects with low coverage and $60 \%$ of subjects with sufficient coverage reported cleaning the syringe before reusing.

\subsection{Model Estimates of Impact (HIV Averted)}

Overall, we estimated the number of new HIV infections due to sharing injections with insufficient NSP coverage as 40.4 per 1000 PWID. Amongst those with sufficient NSP coverage, the HIV infection rate was estimated 
as 10.2 per 1000 IDU. The impact projections are shown in Table 2. The results of the uncertainty analysis showed that NSP with sufficient coverage could avert 30 (95\%SI:15.136.2) new HIV infections per 1000 IDUs per year. In others words, HIV infections prevented by sufficient coverage among PWID is about 75.2\% (95\%SI: 70.4\%-78.1\%).

\subsection{Sensitivity Analysis}

For sensitivity analysis, each input parameter is changed $\pm 1 \mathrm{SD}$, and its effect on effectiveness is calculated and shown by Tornado Plots (Figure 1). This figure represents the possible HIV averted range given the variability around each parameter. Sensitivity analysis by constructing tornado plots demonstrate the robustness of the model in which changing the input parameters \pm 1SD results in less than $0.5 \mathrm{SD}$ in outputs (HIV Averted). The average number of times each shared syringe is used before disposal, average size sharing groups, percent of PWID had sharin and prevalence of HIV amongst PWID were the most behavioral factors associated with NSP effectiveness (Table 2).

Table 2. Uncertainty Analysis for Cumulative Incidence of HIV Infection per 1000 PWID per Year Sufficient/Insufficient Coverage

\begin{tabular}{lccc}
\hline $\begin{array}{l}\text { Outcome } \\
\text { Measure }\end{array}$ & $\begin{array}{c}\text { Insufficient } \\
\text { Coverage Mean } \\
(\text { CI 95\%) }\end{array}$ & $\begin{array}{c}\text { Sufficient } \\
\text { Coverage Mean } \\
(\text { CI 95\%) }\end{array}$ & Difference \\
\hline HIV incidence & $40.4(28.2-56.1)$ & $10.2(3.3-16.4)$ & $30.2(15.1-36.2)$ \\
\hline
\end{tabular}

\section{Discussion}

The analysis used economic analysis and mathematical modeling to estimate the effectiveness of an NSP intervention in Kermanshah, Iran. The results suggest that, despite the high HIV prevalence, NSP's with sufficient coverage can be effectively averted to about 30 new cases of HIV a year per 1000 active PWID, with an even more effect when including their sexual partners. Needle sharing, which is still common amongst injecting drug users (18\%) who are linked to NSP services can be reduced more to $11 \%$, if they have been provided sufficient number of needles and syringes though ongoing NSP activities. Many studies have reported that NSP or NSEP can decrease syringe sharing or HIV transmission but few studies have used mathematical modeling to consider the impact of coverage of NSP on HIV occurrences (31). In present studies, for the first time after implementing and scaling up the NSP in Iran, we used mathematical models in order to estimate HIV infections averted by ensuring sufficient coverage of NSP. This model can also be used to estimate how the expected incidence may change due to changes in syringe distribution through NSPs (30). Worldwide, many studies have reported that NSP or NSEP can decrease HIV incidence or HIV transmission (6, 30, 32-35). Vickerman et al. modeled the impact NSP and showed that by increasing the coverage of NSP, HIV incidences will decrease to $47 \%$ (30). Similar to other studies, we also found that injecting drug users are connected with two to three people who also inject drugs (36). Being provided sufficient syringes and needles ensure that they have enough sterile needles/syringes for their injection; and such connections amongst PWID to be remained safe and reduce the risk of needle sharing and further transmission of infection amongst them. However, needle sharing may occur for social reasons, and not always due to lack of sterile syringes available to them (30). Our study cannot differentiate such effects and so the true effect for increasing the coverage of NSP could be less than what we observed. As previously stated, another limitation to our study is ignoring the indirect effect of increasing the coverage of NSP amongst PWID through reducing the further transmission of HIV to their sexual partners. The impact could be even more than what we observed just for PWID, themselves. Internationally, what is reported as one measure of NSP coverage is the number of new syringes distributed per injector per annum, which is misleading. A more individual based measure, which can truly indicate the gap, is individual NSP converge. Such gap even exists amongst NSP service attendees. This has also been also reported in other studies, even in developing countries like Australia (16). The reasons for this gap are not clear and need to be further investigated. Providing more syringes per visit seems to work, but other reasons should not be neglected. In summary, our analysis highlights that NSP can be effective in a high prevalence setting. However, for harm reduction interventions to substantially improve the epidemic situation, they need to increase their coverage to higher levels than what was attained in Kermanshah, Iran. This could be done by encouraging more frequent visits, increasing hours and locations, and providing more syringes per visit $(20,21,37)$. Although education and counseling is designed to reduce syringe sharing, increasing syringe coverage through NSPs is likely to be the most effective strategy to reduce incident infections. Increasing syringe coverage aims to decrease the number of times each syringe is shared and reduce the frequency of sharing $(38,39)$. Iran has been able to effectively introduce NSPs and increase the coverage of sterile injecting equipment. 


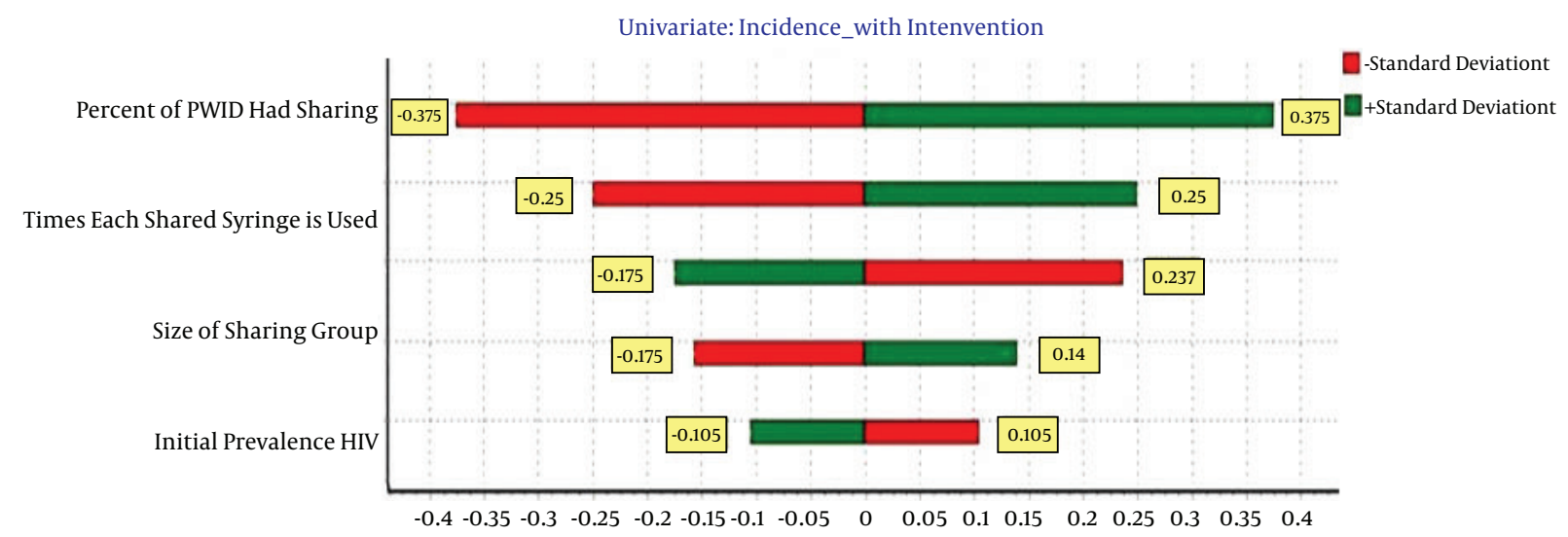

Figure 1. Sensitivity Analysis of HIV Averted for Coverage NSP Program (Tornado Plot)

\section{Acknowledgments}

We gratefully thank the staff of the drop-in centers in Kermanshah, Iran who have contribute in recruiting (Barkhordar) and data collection/interview (Azad and Amini). We profoundly thank everyone who was generous enough to give us their time and participation for this study. We also want to thank experts Mostafa Shokohi and Korosh Etemad from regional knowledge Hub for HIV surveillance who have provided valuable inputs to the study protocol and the questionnaire.

\section{Footnotes}

Authors' Contribution: Study concept and design: Hamid Soori and Mehdi Noroozi; analysis and interpretation of data: Alireza Noroozi and Sayed Saeed Hashemi; drafting the manuscript: Mehdi Noroozi and Ali Mirzazadeh; critical revision of the manuscript: Hamid Sharifi, Ahmad Hajebi, Mehdi Noroozi.

Funding/Support: This article was obtained from a PhD thesis at Shahid Beheshti University of Medical Sciences (SBMU) and funded by Substance abuse prevention and treatment office (SAPTO) of mental health, social health and addiction department (MeHSHAD) of Iran's ministry of health and medical education.

\section{References}

1. United nations offce on drugs and crime . World drug report 2012. Available from: http://www.unodc.org/documents/data-andanalysis/WDR2012/WDR_2012_web_small.pdf.

2. Wilson DP, Kwon A, Anderson J, Thein R, Law MG, Maher L. Return on investment 2: Evaluating the cost-effectiveness of needle and syringe programs in Australia. Australia: Department of Health and Ageing; 2009.
3. Abu-Raddad LJ, Hilmi N, Mumtaz G, Benkirane M, Akala FA, Riedner G, et al. Epidemiology of HIV infection in the Middle East and North Africa. AIDS. 2010;24 Suppl 2:S5-23. doi: 10.1097/01.aids.0000386729.56683.33. [PubMed: 20610949].

4. Nasirian M, Doroudi F, Gooya MM, Sedaghat A, Haghdoost AA. Modeling of human immunodeficiency virus modes of transmission in Iran.J Res Health Sci. 2012;12(2):81-7. [PubMed: 23241516].

5. Khajehkazemi R, Osooli M, Sajadi L, Karamouzian M, Sedaghat A, Fahimfar N, et al. HIV prevalence and risk behaviours among people who inject drugs in Iran: the 2010 National Surveillance Survey. Sex Transm Infect. 2013;89 Suppl 3:iii29-32. doi: 10.1136/sextrans-2013051204. [PubMed: 24037249].

6. Shrestha S, Smith MW, Broman KW, Farzadegan H, Vlahov D, Strathdee SA. Multiperson use of syringes among injection drug users in a needle exchange program: a gene-based molecular epidemiologic analysis. J Acquir Immune Defic Syndr. 2006;43(3):335-43. doi: 10.1097/01.qai.0000230528.25083.0b. [PubMed:16980914].

7. Mirahmadizadeh AR, Majdzadeh R, Mohammad K, Forouzanfar MH. Prevalence of HIV and hepatitis $C$ virus infections and related behavioral determinants among injecting drug users of drop-in centers in Iran. Iran Red Crescent Med J. 2009;11(3):325.

8. Vazirian M, Nassirimanesh B, Zamani S, Ono-Kihara M, Kihara M, Ravari SM, et al. Needle and syringe sharing practices of injecting drug users participating in an outreach HIV prevention program in Tehran, Iran: a cross-sectional study. Harm Reduct J. 2005;2:19. doi: 10.1186/1477-7517-2-19. [PubMed: 16212655].

9. Noroozi M, Mirzazadeh A, Noroozi A, Mehrabi Y, Hajebi A, Zamani S, et al. Client-level coverage of needle and syringe program and highrisk injection behaviors: a case study of people who inject drugs in Kermanshah, Iran. Addict Health. 2015;7(3-4):164.

10. Zamani S, Vazirian M, Nassirimanesh B, Razzaghi EM, Ono-Kihara M, Mortazavi Ravari S, et al. Needle and syringe sharing practices among injecting drug users in Tehran: a comparison of two neighborhoods, one with and one without a needle and syringe program. AIDS Behav. 2010;14(4):885-90. doi: 10.1007/s10461-008-9404-2. [PubMed: 18483849].

11. World Health Organization . Towards universal access: scaling up priority HIV/AIDS interventions in the health sector: progress report 2010. Geneva: World Health Organization; 2010.

12. Mathers BM, Degenhardt L, Ali H, Wiessing L, Hickman M, Mattick RP, et al. HIV prevention, treatment, and care services for people who inject drugs: a systematic review of global, regional, and national coverage. Lancet. 2010;375(9719):1014-28. doi: 10.1016/S0140-6736(10)602322. [PubMed: 20189638]. 
13. Strathdee SA, Patrick DM, Currie SL, Cornelisse PG, Rekart ML, Montaner JS, et al. Needle exchange is not enough: lessons from the Vancouver injecting drug use study. AIDS. 1997;11(8):F59-65. [PubMed: 9223727].

14. Wodak A, McLeod L. The role of harm reduction in controlling HIV among injecting drug users. AIDS. 2008;22 Suppl 2:S81-92. doi: 10.1097/01.aids.0000327439.20914.33. [PubMed: 18641473].

15. Salazar C, Hamidreza S. Uniting the World Against AIDS. Iran (Islamic Republic of). Joint United Nations Programme on HIV, AIDS. UNAIDS; 2006.

16. Iversen J, Topp L, Wand $\mathrm{H}$, Maher $\mathrm{L}$. Individual-level syringe coverage among Needle and Syringe Program attendees in Australia. Drug Alcohol Depend. 2012;122(3):195-200. doi: 10.1016/j.drugalcdep.2011.09.030. [PubMed: 22071120].

17. Zhang L, Yap L, Xun Z, Wu Z, Wilson DP. Needle and syringe programs in Yunnan, China yield health and financial return. BMC Public Health. 2011;11:250. doi: 10.1186/1471-2458-11-250. [PubMed: 21507267].

18. Rhodes T, Hedrich D. Harm reduction: evidence, impacts and challenges. Office for Official Publ. of the European Communities; 2010.

19. Mathers BM, Degenhardt L, Phillips B, Wiessing L, Hickman M, Strathdee SA, et al. Global epidemiology of injecting drug use and HIV among people who inject drugs: a systematic review. Lancet. 2008;372(9651):1733-45. doi: 10.1016/S0140-6736(08)61311-2. [PubMed: 18817968].

20. Bluthenthal RN, Ridgeway G, Schell T, Anderson R, Flynn NM, Kral $\mathrm{AH}$. Examination of the association between syringe exchange program (SEP) dispensation policy and SEP client-level syringe coverage among injection drug users. Addiction. 2007;102(4):638-46. doi: 10.1111/j.1360-0443.2006.01741.x. [PubMed: 17286637].

21. Bluthenthal RN, Anderson R, Flynn NM, Kral AH. Higher syringe coverage is associated with lower odds of HIV risk and does not increase unsafe syringe disposal among syringe exchange program clients. Drug Alcohol Depend. 2007;89(2-3):214-22. doi: 10.1016/j.drugalcdep.2006.12.035. [PubMed:17280802].

22. Patel P, Borkowf CB, Brooks JT, Lasry A, Lansky A, Mermin J. Estimating per-act HIV transmission risk: a systematic review. AIDS. 2014;28(10):1509-19. doi: 10.1097/QAD.0000000000000298. [PubMed: 24809629].

23. Hudgens MG, Longini IJ, Vanichseni S, Hu DJ, Kitayaporn D, Mock PA, et al. Subtype-specific transmission probabilities for human immunodeficiency virus type 1 among injecting drug users in Bangkok, Thailand. Am J Epidemiol. 2002;155(2):159-68. [PubMed: 11790680].

24. Kaplan EH, Heimer R. A model-based estimate of HIV infectivity via needle sharing. J Acquir Immune Defic Syndr. 1992;5(11):1116-8. [PubMed: 1403641].

25. Abdala N, Crowe M, Tolstov Y, Heimer R. Survival of human immunodeficiency virus type 1 after rinsing injection syringes with different cleaning solutions. Subst Use Misuse. 2004;39(4):581-600. [PubMed: 15115214].

26. Abdala N, Gleghorn AA, Carney JM, Heimer R. Can HIV-1-contaminated syringes be disinfected? Implications for transmission among injection drug users. J Acquir Immune Defic Syndr. 2001;28(5):487-94. [PubMed: 11744839]

27. Amin-Esmaeili M, Rahimi-Movaghar A, Razaghi EM, Baghestani
AR, Jafari S. Factors Correlated With Hepatitis C and B Virus Infections Among Injecting Drug Users in Tehran, IR Iran. Hepat Mon. 2012;12(1):23-31. doi: 10.5812/kowsar.1735143X.806. [PubMed 22451840].

28. Jones L, Pickering L, Sumnall H, McVeigh J, Bellis MA. A review of the effectiveness and cost-effectiveness of needle and syringe programmes for injecting drug users. Liverpool: Centre for Public Health; 2008.

29. Vickerman P, Kumaranayake L, Balakireva O, Guinness L, Artyukh O, Semikop T, et al. The cost-effectiveness of expanding harm reduction activities for injecting drug users in Odessa, Ukraine. Sex Transm Dis. 2006;33(10 Suppl):S89-102. doi: 10.1097/01.olq.0000221335.80508.fa. [PubMed: 16735956].

30. Vickerman P, Hickman M, Rhodes T, Watts C. Model projections on the required coverage of syringe distribution to prevent HIV epidemics among injecting drug users. J Acquir Immune Defic Syndr. 2006;42(3):355-61. doi:10.1097/01.qai.0000219788.73539.47. [PubMed: 16645549].

31. Gibson DR, Flynn NM, Perales D. Effectiveness of syringe exchange programs in reducing HIV risk behavior and HIV seroconversion among injecting drug users. AIDS. 2001;15(11):1329-41. [PubMed: 11504954].

32. Schumacher JE, Fischer G, Qian HZ. Policy drives harm reduction for drug abuse and HIV/AIDS prevention in some developing countries. Drug Alcohol Depend. 2007;91(2-3):300-5. [PubMed: 18038459].

33. Wodak A, Cooney A. Effectiveness of sterile needle and syringe programmes. Int J Drug Policy. 2005;16:31-44

34. Wodak A, Cooney A. Do needle syringe programs reduce HIV infection among injecting drug users: a comprehensive review of the international evidence. Subst Use Misuse. 2006;41(6-7):777-813. doi: 10.1080/10826080600669579. [PubMed: 16809167].

35. Kumaranayake L, Vickerman P, Walker D, Samoshkin S, Romantzov V, Emelyanova Z, et al. The cost-effectiveness of HIV preventive measures among injecting drug users in Svetlogorsk, Belarus. Addiction. 2004;99(12):1565-76. doi: 10.1111/j.1360-0443.2004.00899.x. [PubMed: 15585048]

36. Des Jarlais DC, Perlis T, Arasteh K, Hagan H, Milliken J, Braine N, et al "Informed altruism" and "partner restriction" in the reduction of HIV infection in injecting drug users entering detoxification treatment in New York City, 1990-2001. J Acquir Immune Defic Syndr. 2004;35(2):15866. [PubMed: 14722449].

37. Supreme Council of Nationwide Planning of HIV/AIDS Infection Prevention and Control . Monitoring the Declaration of Commitment Adopted by the United Nations General Assembly Special Session on HIV/AID. ; 2014.

38. Kwon JA, Iversen J, Maher L, Law MG, Wilson DP. The impact of needle and syringe programs on HIV and HCV transmissions in injecting drug users in Australia: a model-based analysis. J Acquir Immune Defic Syndr. 2009;51(4):462-9. doi:10.1097/QAI.0b013e3181a2539a. [PubMed: 19387355].

39. Sarang A, Rhodes T, Platt L. Access to syringes in three Russian cities: implications for syringe distribution and coverage. Int J Drug Policy. 2008;19 Suppl 1:S25-36. doi: 10.1016/j.drugpo.2007.11.008. [PubMed: 18313910]. 